\title{
Composición corporal y somatotipo de nadadores adolescentes federados
}

\author{
José Miguel Martínez-Sanz ${ }^{\mathrm{a}, \mathrm{b}, *}$, Juan Mielgo-Ayuso ${ }^{c}$ y Aritz Urdampilleta ${ }^{\mathrm{b}, \mathrm{d}, \mathrm{e}}$ \\ a Programa de Tecnificación de Triatlón, Universidad de Alicante, Alicante, España \\ ${ }^{\mathrm{b}}$ Asesoramiento científico-técnico para la planificación deportiva, NUTRIAKTIVE, España \\ 'Centro Riojano de Nutrición Haro, Club Vóleibol Haro, Haro, La Rioja, España \\ ${ }^{\mathrm{d} D e p a r t a m e n t o}$ de Educación Física y Deportiva, Universidad del País Vasco/Euskal Herriko Unibertsitatea (UPV-EHU), \\ Vitoria, Álava, España \\ eAsesoría Nutricional y Deportiva, Unidad de Fisiología del Deporte, Estímulos de Hipoxia Intermitente y Entrenamientos \\ en Altitud, Centro Deportivo K2, Vitoria-Gasteiz, Álava, España
}

Recibido el 28 de agosto de 2012; aceptado el 30 de octubre de 2012

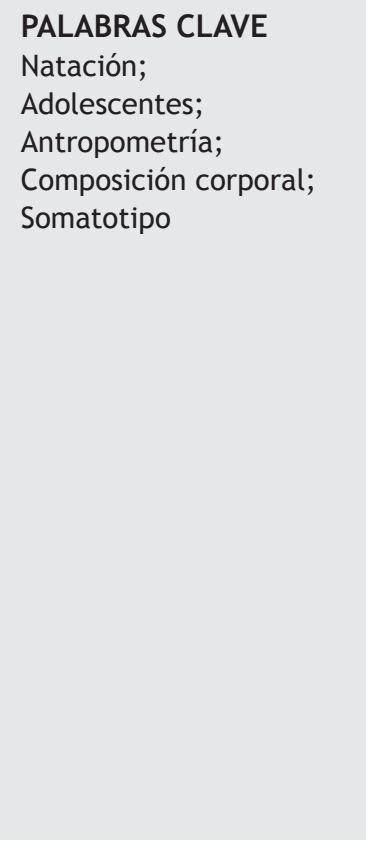

\begin{abstract}
Resumen
Introducción: El nadador es un deportista alto, con extremidades largas, cuyos componentes muscular y óseo son importantes en el éxito deportivo; en cambio, el componente graso resulta paradójico por su relación con la flotabilidad y el desplazamiento del cuerpo en el agua. El objetivo del estudio es describir el perfil antropométrico de nadadores adolescentes de competición.

Material y métodos: Se evaluó a 17 nadadores adolescentes, 10 varones (13,2 $\pm 1,32$ años) y 7 mujeres ( $15 \pm 1,83$ años). Un antropometrista acreditado de nivel 2 tomó las medidas según el protocolo antropométrico de la Society for the Advancement of Kinanthropometry (ISAK), con material homologado según la metodología de Marfell-Jones et al. Se calculó la composición corporal según el consenso de cineantropometría y el somatotipo según Heath-Carter.

Resultados: De todas las variables estudiadas (peso, talla, pliegues, perímetros, diámetros y longitudes), se han hallado diferencias significativas $(p<0,05)$ por sexo en: talla, envergadura, pliegues cutáneos (tricipital, subescapular, cresta iliaca, ileoespinal, abdominal, muslo, pierna y sumatorio de pliegues), índice biacromio-bicrestal, masa corporal ósea, muscular y grasa, endomorfia y ectomorfia.

Conclusiones: Existen diferencias antropométricas significativas entre los nadadores y las nadadoras adolescentes $(p<0,05)$. Dichas diferencias, resultantes del desarrollo de cada sexo, podrían estar muy relacionadas con el rendimiento deportivo de los jóvenes atletas. (c) 2012 Asociación Española de Dietistas-Nutricionistas. Publicado por Elsevier España, S.L. Todos los derechos reservados.
\end{abstract}

*Autor para correspondencia.

Correo electrónico: josemiguel.ms@ua.es (J.M. Martínez). 


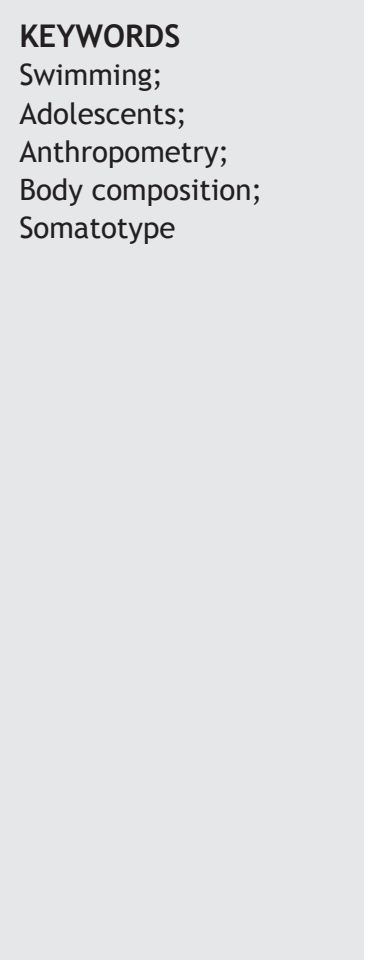

\section{Body Composition and Somatotype in Adolescent Competion Swimmers}

\begin{abstract}
Introduction: The swimmer is a high level athlete, with long limbs, and whose musculoskeletal components are important in sporting success. However, the fat component is paradoxical because of its relationship with the buoyancy and displacement of the body in the water. The aim of this study is to describe the anthropometric profile of adolescent competition swimmers.

Material and methods: A total of 17 adolescent swimmers were evaluated, 10 boys (13.2 \pm 1.32 years) and 7 girls $(15 \pm 1.83$ years $)$. A qualified anthropometrist took anthropometric measures according to the protocol of the Society for theAdvancement of Kinanthropometry (ISAK), with approved materials according to the methodology of Marfell-Jones et al, 2003. Body composition was calculated according to the consensus of Kinanthropometry and somatotype with Heath-Carter's method.

Results: Of all the variables studied (weight, height, folds, circumferences, diameters and lengths), significant differences were found $(P<.05)$ between the sexes in height, arm span, skinfolds (triceps, subscapular, crestailiaca, ileo-spinal, abdominal, thigh, leg, sum of skinfolds), biacromial-bi-iliac index, bone body mass, muscle and fat, ectomorphy, and endomorphy.

Conclusions: There are significant anthropometric differences between genders in adolescent swimmers $(P<.05)$. Such differences, resulting in the development of both sexes, might be highly related to athletic performance of young athletes.

(c) 2012 Asociación Española de Dietistas-Nutricionistas. Published by Elsevier España, S.L. All rights reserved.
\end{abstract}

\section{Introducción}

La antropometría es una de las ciencias aplicadas al deporte que tiene mayor importancia en la evaluación de los deportistas. Ross et $\mathrm{al}^{1}$ (1980) definieron la cineantropometría como "el área de la aplicación del estudio del tamaño, forma, proporción, composición, maduración y funciones principales del ser humano". La antropometría nos ayuda a entender las posibilidades del movimiento humano en el contexto del crecimiento, el ejercicio, el rendimiento y la nutrición. La cineantropometría del deporte posibilita la valoración de las características morfológicas, así como su control durante toda la temporada deportiva. La técnica antropométrica nos permite medir el peso corporal, la estatura y diferentes longitudes, diámetros, perímetros y pliegues cutáneos. Mediante los datos obtenidos, y gracias a la aplicación de diferentes fórmulas, se puede obtener información sobre el somatotipo, la composición corporal y la proporcionalidad de diferentes partes del cuerpo ${ }^{2}$.

Los cuerpos de los deportistas que participan en diferentes deportes o modalidades difieren tanto en tamaño como en composición y forma, tal y como indican Carter $^{3}$ y Eiben ${ }^{4}$.

Las características cineantropométricas del nadador son: deportista alto, ligero, de hombros anchos (diámetro biacromial) y extremidades muy largas. Dichas características potencian la fuerza que ejercen las extremidades superiores y las manos. Los varones poseen un somatotipo medio ectomesomorfo, mientras que las mujeres lo tienen endomesomorfo ${ }^{5}$.
Los factores antropométricos que determinan el rendimiento en natación son la estatura, la envergadura, el peso y la longitud de las extremidades, sobre todo las manos y los pies ${ }^{6-9}$. Carter et $\mathrm{al}^{10}$ establecieron los siguientes perfiles antropométricos para varones y mujeres, respectivamente, que practican estilo de crol: estatura $(186$ y $174 \mathrm{~cm})$, peso (78 y $63 \mathrm{~kg})$, envergadura (194 y $179 \mathrm{~cm})$, longitud de la mano $(21$ y $19,5 \mathrm{~cm})$ y longitud del pie $(27,5$ y $26 \mathrm{~cm})$.

La composición corporal es imprescindible para definir el morfotipo de los nadadores de alto rendimiento. El porcentaje de la masa muscular y ósea resulta de gran interés, mientras que el componente graso es menos importante. Paradójicamente, a medida que aumenta el porcentaje graso, se favorece la flotabilidad y el desplazamiento del cuerpo en el agua, pues se beneficia la hidrodinámica por reducción del área de contacto de la superficie corporal y el gasto metabólico de traslación $n^{5,11}$.

De cada uno de los elementos de la composición corporal, no existe un valor determinante que por sí solo determine el éxito deportivo, sino que el valor conjunto de todos los componentes diferenciaría a los mejores nadadores ${ }^{5,12}$.

Cabe destacar el estudio del dimorfismo sexual en natación, pues hay notorias diferencias antropométricas entre los sexos. Debido a la distribución ginoide del tejido adiposo en las mujeres, podría modificarse la flotabilidad y reducirse la resistencia hidrodinámica al desplazamiento en el agua. En este sentido, se han descrito diferencias relevantes en parámetros como la talla, el peso y las masas grasa, muscular y ósea. En un estudio de revisión sobre la composición corporal y el somatotipo en deportistas españoles ${ }^{13}$, 
entre los adolescentes se hallaron valores de grasa corporal de un $10-11 \%$ en los varones y un $24-28 \%$ en las mujeres (estimación según la fórmula de Faulkner en varones ${ }^{14}$ y Carter en mujeres ${ }^{15}$ ). Además, las nadadoras adolescentes mostraron un valor de $73 \mathrm{~mm}$ en el sumatorio de seis pliegues (tricipital + subescapular + supraespinal + abdominal + muslo + pierna), valor que indica un aumento de masa gra$\mathrm{sa}^{11,16,17}$. Asimismo, el somatotipo es muy heterogéneo entre los sexos, ya que en los varones predomina el ectomesomorfo y el mesomorfo balanceados; en cambio, en las mujeres predomina el endomesomorfo ${ }^{5}$.

El objetivo del estudio es describir el perfil cineantropométrico de nadadores adolescentes federados en la Región de Murcia y establecer las posibles diferencias entre sexos.

\section{Material y métodos}

Estudio observacional y descriptivo de las características cineantropométricas de la composición corporal y el somatotipo.

\section{Muestra}

Se evaluó a 17 nadadores, 10 varones (13,2 $\pm 1,32$ años) y 7 mujeres ( $15 \pm 1,83$ años), federados en la Región de Murcia, con un tiempo de experiencia deportiva entre 3 y 5 años. Todos los deportistas, así como sus respectivos padres o tutores legales, fueron informados sobre las características del estudio. Antes de iniciarse el estudio, todos firmaron el consentimiento informado.

\section{Instrumentos y procedimiento}

Para la valoración cineantropométrica, se siguieron las normas y técnicas de medición recomendadas por el International Working Group of Kinanthropometry, según metodología descrita por Ross et al ${ }^{18}$ en 1991 y adoptadas por la ISAK ${ }^{19}$ y el Grupo Español de Cineantropometría (GREC) ${ }^{20}$.

Se utilizó el siguiente material antropométrico: a) tallímetro de pared (precisión, $1 \mathrm{~mm}$ ); b) báscula (precisión, $100 \mathrm{~g})$; c) cinta métrica Rosscraft metálica, estrecha e inextensible (precisión, $1 \mathrm{~mm}$ ); d) paquímetro de diámetros óseos pequeños Holtain (precisión, $1 \mathrm{~mm}$ ); e) paquímetro de diámetros óseos grandes Holtain (precisión, $1 \mathrm{~mm}$ ); f) plicómetro Holtain (precisión, 0,2 mm), y g) material complementario (lápiz dermográfico para marcar al sujeto).

Un medidor acreditado ISAK de nivel II tomó las siguientes medidas necesarias para la valoración antropométrica, teniendo en cuenta el error técnico de medición (ETM) intraobservador indicado por la ISAK (2001) para nivel II (el 7,5\% para pliegues y el 1\% para perímetros y diámetros): a) pliegues cutáneos (subescapular, tricipital, bicipital, ileocrestal, ileoespinal, abdominal, muslo anterior y pantorrilla); b) perímetros (brazo relajado, brazo contraído, cintura, cadera, muslo y pantorrilla); c) diámetros pequeños (húmero, muñeca y fémur) y diámetros grandes (biacromial y biileocrestal).

Mediante las fórmulas descritas en el consenso de cineantropometría del GREC ${ }^{21}$, se calculó la composición cor- poral mediante el modelo de cuatro componentes: a) masa grasa mediante la ecuación de Withers ${ }^{22,23}$, Carter $^{15}$ y Faulkner ${ }^{14}$; b) masa muscular mediante la propuesta de Lee et $\mathrm{al}^{24}$, y c) masa ósea mediante la de Rocha ${ }^{25}$. También se calculó el sumatorio de ocho pliegues (subescapular, tricipital, bicipital, ileocrestal, ileoespinal, abdominal, muslo anterior y pantorrilla), el índice de distribución de la grasa (IDG) como (subescapular + tricipital + bicipital + abdominal) / (ileocrestal + ileoespinal + muslo anterior + pantorrilla) y el índice biacromio-biileocrestal (diámetro biacromial / diámetro biileocrestal).

Para el cálculo del somatotipo, se determinó el somatotipo medio, los tres componentes del somatotipo por separado (endomorfia, mesomorfia, ectomorfia) y la distancia morfogenética del somatotipo (SAD), siguiendo el método de Heath-Carter ${ }^{26,27}$. Todos los parámetros se determinaron y clasificaron según sexo.

\section{Análisis estadístico}

Todos los datos se presentan como media \pm desviación estándar. Se comparó cada una de las variables entre ambos sexos mediante el test de la t de Student o la U de MannWhitney, en función de que la distribución de las muestras fuera normal o no. La distribución de las muestras se evaluó mediante el test de Shapiro-Wilks. Se consideró diferencia estadísticamente significativa si $p<0,05$. Los análisis estadísticos se realizaron mediante el paquete estadístico SPSS versión 17.0 y la hoja de cálculo Microsoft Excel 2010.

\section{Resultados}

Los resultados obtenidos sobre las características cineantropométricas de la composición corporal se muestran en la tabla 1.

De las características cineantropométricas de la muestra estudiada, se puede destacar:

- Medidas básicas y principales longitudes y diámetros: en conjunto, los nadadores eran altos para su edad (por encima del percentil 90) y las nadadoras no se encontraban por debajo del percentil 50. Más de la mitad de los varones están por encima del percentil 75. En cuanto al peso, las chicas se centran entre p50 y p75, mientras que los varones están por encima de $\mathrm{p} 90$.

- Pliegues cutáneos: se expone el valor de los ocho pliegues cutáneos medidos y la suma de todos ellos. Para obtener el valor del pliegue, se ha tomado la media si se tomaron dos mediciones no consecutivas o la mediana si se tomaron tres no consecutivas de los pliegues, tal y como propone el protocolo ISAK ${ }^{19}$.

- Composición corporal: muestra el cálculo de la composición corporal mediante el modelo de cuatro componentes descrito por el GREC ${ }^{21}$ y el IDG, que muestra que la cantidad de grasa relativa es mayor en las extremidades que en el tronco para ambos sexos.

- Somatotipo: muestra los valores de somatotipo y SAD, con diferencia significativa entre los varones y las mujeres de la muestra estudiada. 
Tabla 1 Características cineantropométricas de la muestra

\begin{tabular}{|c|c|c|c|c|}
\hline & Total $(n=17)$ & Mujeres $(n=7)$ & Varones $(n=10)$ & $\mathrm{p}$ \\
\hline \multicolumn{5}{|l|}{$\begin{array}{l}\text { 1. Medidas básicas y principales longitudes } \\
\text { y diámetros }\end{array}$} \\
\hline Edad & $13,94 \pm 1,75$ & $15,00 \pm 1,83$ & $13,20 \pm 1,32$ & 0,032 \\
\hline Peso (kg) & $59,51 \pm 8,70$ & $56,31 \pm 6,76$ & $61,75 \pm 9,52$ & 0,215 \\
\hline Talla $(\mathrm{cm})$ & $170,18 \pm 9,00$ & $164,29 \pm 4,63$ & $174,30 \pm 9,15$ & 0,018 \\
\hline IMC & $20,46 \pm 1,63$ & $20,80 \pm 1,59$ & $20,22 \pm 1,69$ & 0,486 \\
\hline Envergadura $(\mathrm{cm})$ & $173,57 \pm 11,05$ & $166,39 \pm 7,61$ & $178,60 \pm 10,50$ & 0,019 \\
\hline Diámetro biacromial & $36,24 \pm 3,01$ & $34,31 \pm 2,10$ & $37,59 \pm 2,87$ & 0,021 \\
\hline Diámetro biiliocrestal & $27,82 \pm 1,59$ & $28,36 \pm 2,07$ & $27,45 \pm 1,11$ & 0,259 \\
\hline Índice biacromio-bicrestal & $1,31 \pm 0,12$ & $1,21 \pm 0,09$ & $1,37 \pm 0,10$ & 0,005 \\
\hline Longitud de la mano & $17,84 \pm 1,31$ & $17,06 \pm 1,30$ & $18,39 \pm 1,06$ & 0,034 \\
\hline Longitud del fémur & $39,37 \pm 2,66$ & $38,26 \pm 2,00$ & $40,15 \pm 2,87$ & 0,154 \\
\hline \multicolumn{5}{|l|}{ 2. Valores de pliegues cutáneos } \\
\hline Subescapular & $10,02 \pm 2,32$ & $11,57 \pm 2,47$ & $8,93 \pm 1,52$ & 0,015 \\
\hline Tricipital & $11,15 \pm 4,18$ & $14,04 \pm 4,21$ & $9,12 \pm 2,86$ & 0,011 \\
\hline Bicipital & $6,18 \pm 2,51$ & $7,83 \pm 3,03$ & $5,02 \pm 1,22$ & 0,510 \\
\hline Cresta iliaca & $14,98 \pm 5,32$ & $18,43 \pm 4,10$ & $12,57 \pm 4,84$ & 0,020 \\
\hline Supraespinal & $10,27 \pm 4,32$ & $12,71 \pm 4,86$ & $8,56 \pm 3,09$ & 0,047 \\
\hline Abdominal & $16,16 \pm 5,60$ & $19,77 \pm 4,90$ & $13,64 \pm 4,74$ & 0,021 \\
\hline Muslo medio & $15,41 \pm 4,70$ & $19,07 \pm 4,11$ & $12,85 \pm 3,22$ & 0,003 \\
\hline Pierna & $11,91 \pm 4,55$ & $15,69 \pm 3,67$ & $9,26 \pm 3,01$ & 0,001 \\
\hline Sumatorio 8 pliegues & $96,08 \pm 30,24$ & $119,11 \pm 25,62$ & $79,95 \pm 22,06$ & 0,004 \\
\hline \multicolumn{5}{|l|}{ 3. Composición corporal } \\
\hline Peso muscular Lee (\%) & $43,00 \pm 4,19$ & $38,72 \pm 2,54$ & $46,00 \pm 1,64$ & $<0,001$ \\
\hline Peso óseo Rocha (\%) & $17,61 \pm 2,06$ & $16,20 \pm 1,29$ & $18,60 \pm 1,95$ & 0,013 \\
\hline Peso graso Whiters (\%) & $12,78 \pm 3,31$ & $14,12 \pm 3,40$ & $11,84 \pm 3,07$ & 0,170 \\
\hline Grasa Faulkner (\%) & $15,37 \pm 4,73$ & $20,28 \pm 2,78$ & $11,94 \pm 1,69$ & $<0,001$ \\
\hline Grasa Carter (\%) & $12,77 \pm 4,99$ & $17,95 \pm 2,94$ & $9,14 \pm 1,73$ & $<0,001$ \\
\hline Índice de distribución de grasa (IDG) & $1,07 \pm 0,21$ & $1,14 \pm 0,27$ & $1,02 \pm 0,16$ & 0,253 \\
\hline \multicolumn{5}{|l|}{ 4. Somatotipo y $S A D$} \\
\hline Endomorfia & $3,19 \pm 1,05$ & $4,02 \pm 0,81$ & $2,61 \pm 0,79$ & 0,003 \\
\hline Mesomorfia & $3,50 \pm 0,55$ & $3,39 \pm 0,41$ & $3,57 \pm 0,65$ & 0,535 \\
\hline Ectomorfia & $3,41 \pm 0,93$ & $2,86 \pm 0,68$ & $3,79 \pm 0,91$ & 0,036 \\
\hline SAD & $1,05 \pm 0,57$ & $0,91 \pm 0,54$ & $1,15 \pm 0,60$ & 0,404 \\
\hline
\end{tabular}

Las figuras 1 y 2 muestran la distribución del somatotipo en chicas y chicos respectivamente. El somatotipo medio se ha marcado con un círculo negro en ambos sexos. El de los varones de la muestra se caracteriza por ser ectomorfomesomorfo, con una distribución ectomorfo-mesomorfo y ectomorfo balanceado en la muestra. Las mujeres presentan una distribución media de endomorfo-mesomorfo, pero se observan somatotipos endomorfo balanceados, central y endomorfo-mesomorfo.

\section{Discusión}

De los resultados obtenidos respecto a las características cineantropométricas de la composición corporal y el somatotipo en la muestra estudiada, se puede extraer los siguientes datos e interpretaciones.

Respecto a la talla y el peso de la muestra, observamos diferencias significativas entre sexos, los varones son más altos y de mayor peso (mayor masa muscular y ósea). Los nadadores estudiados muestran valores similares que otros estudios realizados sobre nadadores olímpicos de edades similares ${ }^{28}$, y estos adolescentes son físicamente más grandes en comparación con chicos y chicas de su misma edad que no compiten ${ }^{28}$. Estudios recientes realizados en nadadores jóvenes ${ }^{29}$ presentan datos de talla y peso muy similares a los expuestos en este estudio, a pesar de que la edad de los chicos de nuestra muestra es 1,3 años menor $(13,2$ frente a 15,5 años), mientras que la edad de las chicas es similar en ambos estudios (15 frente a 14,6 años). Richardson et $\mathrm{al}^{30}$ también mostraron valores semejantes de talla y peso en mujeres de 13-14 años, pero los valores de los chicos eran inferiores $(167,1 \pm 4,3 \mathrm{~cm} ; 54,1 \pm 5,6 \mathrm{~kg})$ a los del presente estudio.

En la composición corporal, los varones presentan mayor desarrollo osteomuscular (mayor masa muscular y ósea) que las mujeres. Respecto al componente graso, es mayor en ellas, tal y como muestra la literatura ${ }^{5}$. Se estimó la masa 


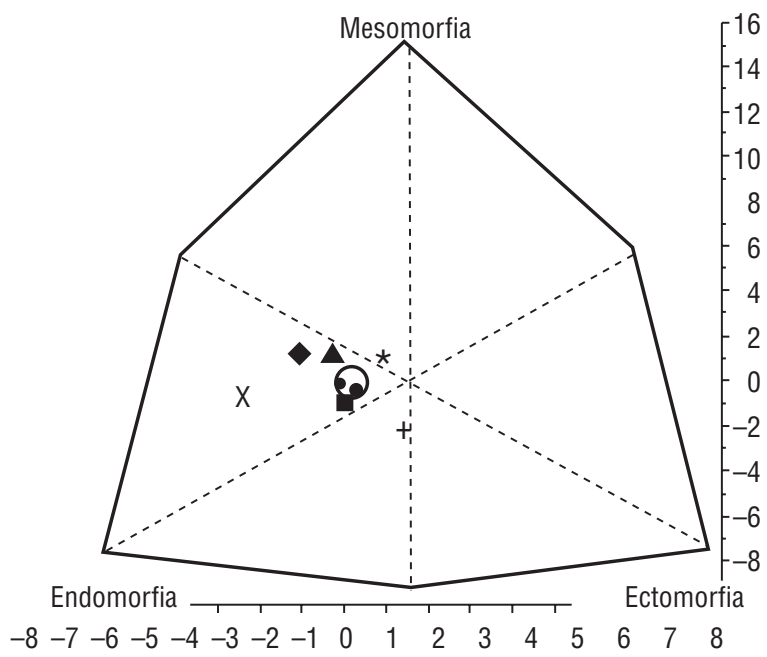

Figura 1 Distribución del somatotipo entre las nadadoras.

grasa utilizando diferentes ecuaciones, debido a que en la literatura se describen estudios que han utilizado la ecuación de Faulkner o Yuhasz ${ }^{29}$ o Carter $^{13}$, pero no hemos encontrado ninguna referencia que haya utilizado la ecuación de Withers, pese a que es una de las recomendadas por el GREC ${ }^{21}$. Observamos que el valor medio del porcentaje graso en ambos sexos se encuentra dentro de la franja de referencia nacional-internacional según Carter $^{31}$. A la vez, los valores obtenidos del porcentaje graso de los varones utilizando tanto la ecuación de Withers como la de Faulkner son similares a los mostrados de nadadores junior extranjeros ${ }^{5}$, nadadores del programa de tecnificación gallego $(11,62$ $12,23 \%$ ) y los obtenidos por Martínez et al ${ }^{29}$ en nadadores españoles. No ocurre lo mismo con los valores de las nadadoras, con valores mucho mayores que los de las nadadoras extranjeras $(12,1 \%)$ con independencia de la ecuación utilizada, las del estudio de Martínez et $a^{29}$ y las del programa de tecnificación gallego ${ }^{32}$; sólo si utilizamos la ecuación de Whiters nuestros datos estarían en la franja baja del estudio gallego (un 14,29-16,75\% en las mujeres), no así con los valores obtenidos mediante las ecuaciones de Faulkner y Carter, que arrojan valores mayores.

En el caso de las mujeres, los valores de nuestro estudio son también mayores que los descritos en otros estudios con las ecuaciones de $\operatorname{Carter}^{13,29,30}$. Ello puede deberse a que nuestra muestra de mujeres era mucho menor en número, a la influencia de otros factores externos (sociales, alimentarios, entrenamiento y ambientales, entre otros) o al grado de maduración sexual, no tenidos en cuenta en el presente estudio ${ }^{5}$. Esto nos hace pensar que las nadadoras requieran una atención especial a la hora de controlar la grasa corporal, especialmente porque se sabe que en la adolescencia ellas terminan la pubertad y es momento ideal para comparar con referentes internacionales y orientar adecuadamente pautas dietético-nutricionales para conseguir parámetros de los deportistas de alto rendimiento deportivo. No obstante, cabe decir de los datos obtenidos en el estudio y las referencia de la literatura que no se ajustan siempre al mismo número de pliegues (Faulkner utiliza cuatro pliegues; Carter, seis y Withers, siete), y en las mujeres

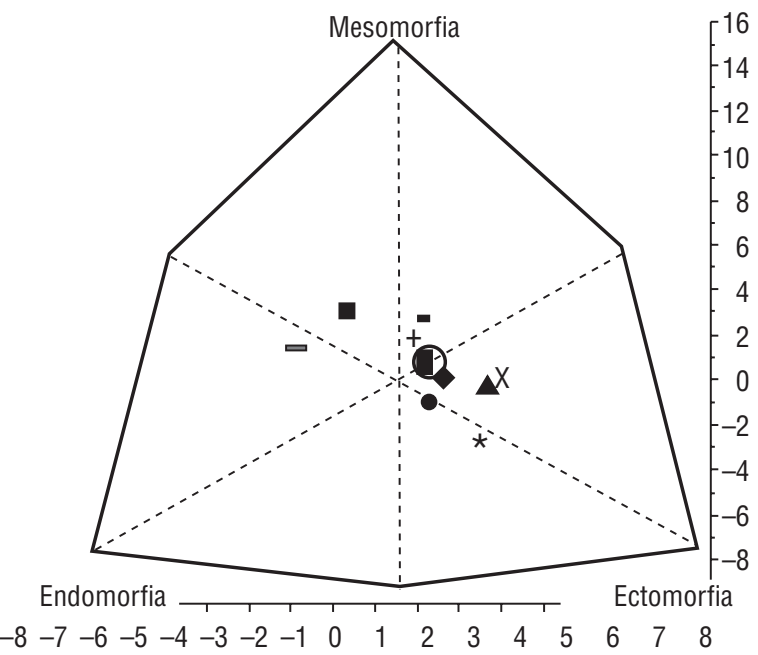

Figura 2 Distribución del somatotipo entre los nadadores.

puede ser más adecuado utilizar la de seis o siete pliegues, ya que recogen datos de los pliegues del tren inferior, en el que las mujeres suelen tener más problemas para perder peso. Por ello se tiende a utilizar el sumatorio de pliegues como indicador de la grasa corporal ${ }^{33}$.

Respecto al componente muscular y óseo, los valores son mayores en los varones; generalmente la masa muscular es un componente que predomina en ellos, y nuestros resultados corroboran las diferencias por sexo. Sólo hemos hallado un estudio realizado sobre 20 nadadores entre 13 y 16 años con el mismo tiempo de entrenamiento (3-5 años $)^{34}$, que muestra valores de masa muscular y ósea (el 46 y el 18,6\% respetivamente) muy similares al del total de los participantes de nuestra muestra (el 43 y el 17,61\%). Valores similares de masa muscular en nadadores gallegos también mostraron Ramirez et al $^{32}$ en 2006 (un 47-49\% en ambos sexos). Hay que considerar estos valores por su relación con el rendimiento deportivo.

Respecto al IDG, los datos obtenidos indican mayor predomino de la grasa relativa en extremidades respecto al tronco, lo que puede deberse a valores altos en pliegues tricipital, bicipital, muslo y pierna. En otros estudios, el grosor de los pliegues de los nadadores es 1-4 mm menor que en nuestra muestra ${ }^{29,35}$.

Los datos de estudios realizados en edades inferiores a 13 años muestran características cineantropométricas diferentes (valores más bajos) en peso, talla y grasa corporal $^{36}$, debido a un estado ponderal y de maduración sexual menor en nuestra muestra, especialmente entre las mujeres.

En el somatotipo, encontramos predominio del componente endomórfico en la mayoría de las mujeres, debido a mayor acumulación de grasa subcutánea, escaso desarrollo muscular y linealidad relativa; este somatotipo es similar al presentado por Martínez et $\mathrm{al}^{29}$ en un estudio con una muestra similar, en el que hallaron en las nadadoras un somatotipo endomórfico balanceado, que es similar al de la población de referencia ${ }^{5}$. Sin embargo, en otros estudios con adultos, el somatotipo de las nadadoras es central o mesomórfico balanceado ${ }^{37}$, con un desarrollo osteomuscular moderado y poca grasa subcutánea. En el caso de los 
nadadores, hay predomino de ectomorfo balanceado y ectomorfo-mesomorfo, con baja adiposidad relativa y mayor desarrollo muscular que en las mujeres. Este somatotipo es muy similar al presentado en estudios con adultos, cuyos componentes ectomórficos y mesomórficos eran los predominantes $^{37}$. Sin embargo, en otros estudios similares ${ }^{29}$ presentaron un somatotipo ectomorfo-endomorfo, por lo que nuestros nadadores tenían mayor desarrollo osteomuscular, mayor linealidad relativa y menor componente adiposo. Los valores de endomorfia de nuestro estudio muestran una moderada adiposidad relativa, principalmente en mujeres, en comparación con otros estudios, cuyos valores de endomorfia son inferiores $(<4)$, con poca grasa subcutánea ${ }^{11,17}$, pero el componente endomórfico es muy parecido en ambos sexos a las referencias de élite internacional junior $^{27}$. Se observó que el componente mesomórfico (valores de 3,5 a 5 para ambos sexos) era mayor en la población de referencia internacional y otros estudios ${ }^{5,11,17}$; el desarrollo osteomuscular es uno de los que determinan el éxito deportivo ${ }^{12}$, por su influencia en la capacidad de generar energía propulsora ${ }^{38}$. De igual forma, se destaca el predominio de la ectomorfia de los nadadores estudiados, que se relaciona con la talla y la linealidad del cuerpo, que implican menor volumen por unidad de estatura y pueden dar explicación a un menor valor del componente mesomórfico ${ }^{5,39}$.

Asimismo, se han hallado diferencias significativas $(p<0,05)$ entre sexos en diferentes parámetros cineantropométricos como edad, talla, envergadura, pliegues cutáneos (tricipital, subescapular, cresta iliaca, ileoespinal, abdominal, muslo, pierna, sumatorio de pliegues), índice biacromio-bicrestal, masas corporales ósea, muscular y grasa, endomorfia y ectomorfia, que se ha de considerar en futuros estudios sobre natación por su influencia en el rendimiento deportivo y las diferencias por sexo ${ }^{29,36}$.

Se debe destacar la importancia del seguimiento de la composición corporal en los estudios con niños y adolescentes. Así, en muestras con idéntica edad cronológica se puede encontrar fases de crecimiento biológico diferentes ${ }^{40}$, de ahí la importancia de estudios adicionales para aclarar la influencia de las etapas de maduración sexual en los parámetros antropométricos y de composición corporal, lo que permitiría la creación de referencias que considerar en el desarrollo de la pubertad. En nuestro caso, la población estudiada se encuentra en periodo de crecimiento puberal en comparación con la de referencia, además de que se encontraban al comienzo de la temporada. De ahí la necesidad de realizar estudios longitudinales para conocer la proporción específica de su composición corporal a lo largo de la temporada y determinar su influencia en el crecimiento y el rendimiento deportivo ${ }^{41,42}$.

\section{Conclusiones}

Los pliegues con mayores diferencias entre sexos son las de tren inferior en pierna $(p=0,001)$ y muslo $(p=0,003)$ y el tren superior en tricipital $(p=0,011)$ y subescapular $(p=0,015)$. El sumatorio de ocho pliegues de las mujeres es de $119,11 \pm 25,62$ y el de los varones, 79,95 $\pm 22,06$ $(p=0,004)$.
En masa grasa, las diferencias son muy significativas en el porcentaje graso (Faulkner) entre los varones y las mujeres, que tienen más porcentaje graso $(p<0,05)$. El valor medio del porcentaje graso (según la ecuación de Faulkner) de los varones $(12,77 \pm 4,99 \%)$ se encuentra en la banda de referencia internacional, aunque el de las mujeres $(17,95 \pm$ $2,94 \%$ ) está ligeramente por encima de las referencias obtenidas en la literatura.

Las diferencias en la masa muscular entre mujeres y varones es muy significativa ( $<<0,001)$, pues ellos tienen más masa muscular $(38,72 \pm 2,54$ frente a $46,00 \pm 1,64)$. La masa muscular de la muestra entera $(43,00 \pm 4,19 \%)$ (fórmula de Lee) es ligeramente inferior a la única muestra hallada de nadadores adolescentes federados (46\%), aunque la masa muscular de los varones $(46,00 \pm 1,64)$ se acerca a los valores de referencia.

En el somatotipo encontramos predominio del componente endomórfico en las mujeres ( $\mathrm{SAD}, 0,91 \pm 0,54)$, aunque en otros estudios han encontrado un somatotipo endomorfo balanceado. En el caso de los varones, hay predomino de ectomorfo balanceado y ectomorfo-mesomorfo (SAD, $1,15 \pm 0,60$ ). Respecto a la muestra, se han encontrado diferencias significativas por sexo en cuanto a la endomorfia $(p=0,003)$ y la ectomorfia $(p=0,03)$, pues los varones tienen menos endomorfia (adiposidad relativa) y más ectomorfia (delgadez-linealidad).

\section{Conflicto de intereses}

Los autores declaran no tener ningún conflicto de intereses.

\section{Bibliografía}

1. Ross WD, Drinkwater DT, Bailey DA, Marshall GH, Leahy RM. Kinantropometry: traditions and new perspectives. Edit. Kinanthropometry II. En: Osting M, Beunen G, Simons J, editores. International series on Sports Sciences. Vol 9. Baltimore: University Partk Press; 1980.

2. Petrosky EL. Antropometría. Técnicas e Paronizações. Porto Alegre Palloti; 2003.

3. Carter JEL. The somatotype of athletes: review. Hum Biol. 1970;42:535-69.

4. Eiben 0 . The physique of women athletes. Budapest: Hungarian Scientific Council Physical Education; 1972.

5. Cabañas Armesilla MD, Esparza Ros F. Compendio de cineantropometria. Madrid: CTO; 2009.

6. Pelayo P, Wille F. Evolution du niveau de practique en natation dans le second degré scolaire français. STAPS. 1994;33: 69-78.

7. Pelayo P. Evaluation, sélection et suivi médico-sportf de nageurs espoirs (11-12 ans). Thèse pour l'obtention du Doctorat S.T.A.P.S., Sciences de la vie et de la santé. Lille: Université de Droit et de la Santé; 1989.

8. Cazorla G, Montpetit R, Fouillot JP, Cervetti JP. Etude méthodologique de la mesure directe de la consommation maximale d'oxygène au cours de la nage. Cinésiologie. 1985;40:21-33.

9. Chatard JC, Padilla S, Cazorla G, Lacour JR. Influence de la morphologie et de l'entraînement sur la performance en natation. STAPS. 1987;8:23-8.

10. Carter JE, Ackland T. Kinanthropometry in aquatic sports. Auckland: Human Kinetics; 1994 
11. Riera J, Javierre C, Ventura JL, Zamora A. Estudio antropométrico y funcional en nadadores. Apunts Medicina de l'Esport. 1994;31:213-31.

12. Pancorbo Sandoval AE. Medicina y ciencias del deporte y actividad física. Consejo Superior de Deportes. Madrid: Ergon; 2008.

13. Fernández Paneque $S$, Alvero Cruz JR. La producción científica en cineantropometría: datos de referencia de composición corporal y somatotipo. Archivos de Medicina del Deporte. 2006;23:17-35.

14. Faulkner J. Physiology of swimming and diving. En: Falls $\mathrm{H}$, editor. Exercise physiology. Baltimore: Academic Press; 1968.

15. Carter J. Body composition of Montreal Olympic athletes. En: Carter J, editor. Physical structure of Olympic athletes Part I. The Montreal Olympic Games Anthropological Project. Basel: Karger; 1982. p. 107-16.

16. López Téllez A, Martí Jiménez A, Martínez Blanco J, Parra Rodríguez JC, Villodres Martí MC, Fernández Alba CF. Antropometría y grado de maduración en nadadores adolescentes. Archivos de Medicina del Deporte. 2002;29:29-35.

17. Palomino Martín A, Ortega Santana F, García Manso JM, Limiñana Cañal JM, Sarmiento Ramos L, Mompeó Corredera B. Estudio cineantropométrico entre nadadores canarios y peninsulares por estilos. Archivos de Medicina del Deporte. 1996;13:433-9.

18. Ross WD, Marfell-Jones MJ. Kinanthropometry. En: MacDougall JD, Wenger HA, Green HJ, editores. Physiological testing of elite athlete. London: Human Kinetics; 1991. p. 223-308.

19. ISAK. International Standards For Anthropometric Assessment. Glasgow: International Society for the Advancement of Kinanthropometry; 2001.

20. Esparza F. Manual de Cineantropometría. Pamplona: GREC-FEMEDE; 1993.

21. Alvero Cruz JR, Cabañas Armesilla MD, Herrero de Lucas A, Martínez Riaza L, Moreno Pascual C, Porta Manceñido J, et al. Protocolo de valoración de la composición corporal para el reconocimiento médico-deportivo. Documento de consenso del Grupo Español de Cineantropometría de la Federación Española de Medicina del Deporte. AMD. 2010;27:330-44.

22. Withers RT, Craig NP, Bourdon PC, Norton KI. Relative body fat and anthropometric prediction of body density of male athletes. Eur J Appl Physiol. 1987;56:191-200.

23. Withers RT, Whittingham NO, Norton KI, La Forgia J, Ellis MW, Crockett A. Relative body fat and anthropometric prediction of body density of female athletes. Eur J Appl Physiol. 1987;56:169-80.

24. Lee R, Wang Z, Heo M, Ross R, Janssen I, Heymsfield S. Totalbody skeletal muscle mass: development and cross-validation of anthropometric prediction models. Am J Clin Nutr. 2000;72:796-803.

25. Rocha MSL. Peso osseo do brasilerio de ambos os sexos de 17 a 25 anos. Arq Anat Antrop. 1975;1:445.
26. Carter JEL. The Heath-Carter somatotype method. San Diego: San Diego State University; 1975.

27. Carter L. Somatotipo. En: Norton K, Olds T, editores. Antrhopometrica. Sydney: University of New South Wales Press; 1996. p. 99-115.

28. Bagnall KM, Kellett DW. A study of potential olympic swimmers: I, the starting-point. Br J Sports Med. 1977;11:127-32.

29. Martinez S, Pasquarelli BN, Romaguera D, Arasa C, Taular $P$, Aguiló A. Anthropometric characteristics and nutritional profile of Young amateur swimmers. J Strength Cond Res. 2001;25:112633.

30. Richardson J, Beerman K, Heiss C, Shultz J. Comparison of body weight and body fat classification of competitive schoolage club swimmers. J Am Diet Assoc. 2000;100:237-40.

31. Canda A. Taller de cineantropometría. Protocolo de medición y valores de referencia. VIII Congreso FEMEDE. Zaragoza, 17-20 noviembre de 1999.

32. Ramirez Farto E, Rivera Lamiguiro J. Plan gallego de tecnificación deportiva: características morfológicas de sus nadadores. Efedeportes. 2006;103.

33. Martínez-Sanz JM, Urdampilleta A. Antropometría y control de peso. Sport Training Magazine. 2012;41:54-7.

34. Enseñat Solé A, Matamala Cura R, Negro Claret A. Estudio antropométrico de nadadores y waterpolistas de 13 a 16 años. Apunts: Educación Física i Esports. 1992;29:12-17.

35. Schneider P, Meyer F. Anthropometric and muscle strength evaluation in prepubescent and pubescent swimmer boys and girls. Rev Bras Med Esporte. 2005;11:200-3.

36. Zuniga J, Housh TJ, Mielke M, Hendrix CR, Camic CL, Johnson $\mathrm{GO}$, et al. Gender comparisons of anthropometric characteristics of young swimmers. J Strength Cond Res. 2011;25:103-8.

37. Fernández R, Barbosa T, Vilas-Boas JP. Determinant kinantropometric factors in swimming. Rev Bras Cineantropom Desempenho Hum. 2002;4:67-79.

38. Maglischo EW. Nadando ainda mais rápido. São Paulo: Manole; 1999.

39. Martínez-Sanz JM, Cejuela R, Cabañas Armesilla MD, Urdampilleta Otegui A. Evaluación cineantropometrica II: Composición corporal, somatotipo y proporcionalidad. Valencia: Universidad de Valencia y Fundación Universidad Empresa ADEIT; 2011.

40. Ferreira Barbosa K, Castro Franceschini S, Priore S. Influence of stages of sexual maturation in the nutritional status, anthropometrics and corporal composition of adolescents. Rev Bras Saúde Matern Infant. 2006;6:375-82.

41. Norton K, Olds T, Olive S, Craig N. Antropometría y performance deportiva. En: Norton K, Olds T, editores. Antrhopometrica. Sydney: University of New South Wales Press; 1996. p. 99-115.

42. Siders WA, Lukaski HC, Bolonchuk WW. Relationships among swimming performance, body composition and somatotype in competitive collegiate swimmers. J Sports Med Phys Fitness. 1993;33:166-71. 